\title{
Article \\ Personalization of a human body model using subject-specific clothing industry dimensions
}

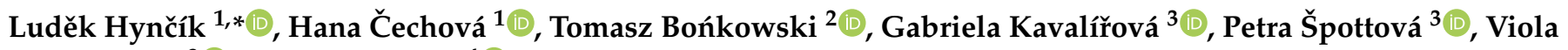 \\ Hampejsová ${ }^{3}\left(\mathbb{C}\right.$ and Hong Meng ${ }^{4}(\mathbb{D})$ \\ 1 New Technologies - Research Centre, University of West Bohemia, Univerzitní 8, 20100 Plzeň, Czech \\ Republic; hyncik@ntc.zcu.cz (L.H.); hcechov@ntc.zcu.cz (H.C.) \\ 2 Faculty of Applied Sciences, University of West Bohemia, Univerzitní 8, 20100 Plzeň, Czech Republic; \\ tomasz@ntc.zcu.cz \\ 3 Faculty of Education, University of West Bohemia, Univerzitní 8, 20100 Plzeň, Czech Republic; \\ kavaliro@ktv.zcu.cz (G.K.); petap@ktv.zcu.cz (P.S.); viola.hampejsova@seznam.cz (V.H.) \\ 4 Tianjin Technician Institute of Mechanical \& Electrical Technology, Yaguan Road 17, Haihe Education Park \\ District, Tianjin, China; 12104918@163.com \\ * Correspondence: hyncik@ntc.zcu.cz, Tel.: +420 377634709 (L.H.)
}

Featured Application: Proving the considerable diversity in anthropometry variations over different population groups, the paper extends the existing scaling algorithm on a virtual biomechanical human body model by a personalization method leading to the development of subject-specific human body models. Such models serve for assessing the injury risk and optimizing personal protective equipment (PPE) for a specific persons using virtual approach and numerical simulation.

\begin{abstract}
Virtual human body models contribute to designing safe and user-friendly products through virtual prototyping. Anthropometric biomechanical models address different physiques using average dimensions. In designing personal protective equipment, biomechanical models with the correct geometry and shape shall play a role. The presented study shows the variations of subject-specific anthropometric dimensions from the average for the different population groups in the Czech Republic and China as a background for the need for personalized human body models. The study measures a set of clothing industry dimensions of Czech children, Czech teens, Czech adults and Chinese adults and compares them to the corresponding age average, which is represented by a scaled anthropometric human body model. The cumulative variation of clothing industry dimensions increases the farer is the population group from the average. It is smallest for the Czech adults $7.54 \% \pm 6.63 \%$, Czech teens report $7.93 \% \pm 6.25 \%$ and Czech children differ $9.52 \% \pm 6.08 \%$. Chinese adults report $10.86 \% \pm 11.11 \%$. As the variations of the particular clothing industry dimensions from the average prove the necessity of having personalized subject-specific models, the personalization of particular body segments using the measured clothing industry dimensions leading to a subject-specific virtual model is addressed. The developed personalization algorithm results in the continuous body surface desired for contact applications for assessing body behavior and injury risk under impact loading.
\end{abstract}

Keywords: human body; anthropometric dimensions; personalization; subject-specific model; biofidelity

\section{Introduction}

Virtual prototyping enables fast product design and optimization using numerical calculation. Virtual human body models play an important role in the design of humanfriendly and safe personal products. Addressing the wide population, customized human body models [1,2] have a wide range of applications. A virtual human body model with biofidelity can also serve for assessing injuries caused by external impacts [3]. 
Such virtual approach towards injury risk assessment is useful for the development of safety systems in mobility addressing autonomous driving, leisure riding, sports activities, sportswear and activewear and many other fields using virtual biomechanical human body models as the optimization and design tasks can be difficult to assess experimentally taking wide populations (EU, US, ASEAN) with varying anthropometry into account. For designing PPE, subject-specific human body models seem like a promising tool for personal approach in safety $[4,5]$.

There are two main steps in generating a customized virtual human body model, namely the scaling and the personalization of a reference model. The scaling procedure implements a reference model and updates its global dimensions to describe an average representative of the population group with the given anthropometry [6-8]. Whilst scaling changes only the global dimensions (anthropometry, mass and stiffness) based on the general anthropometric parameters [6,7], personalization updates the local geometrical and biomechanical details of the particular human body segments [9-11] and so leads to a particular subject-specific model. In general, geometrically the reference model is usually described by a cloud of nodes connected to elements creating the surface mesh. The personalization of such model is usually based on mesh morphing [12], which needs to have a considerable number of corresponding landmarks defined on both the reference and the target models [13,14].

Both approaches are based on a reference model, which is a necessary starting point for the development of a subject-specific model. The scaling approach is simpler as it addresses only global (averaged) anthropometric dimensions for target population groups [7]. Scaling is a perfect tool for creating generic human body models representing a population group for virtual prototyping [8] as they have the correct mass distribution and body flexibility to cover a wide spectrum of the population. However, if we want to address the designing of PPE for a particular person, a subject-specific (personalized) approach is needed as scaling does not provide details of the particular subject even inside a particular population group. Additionally, the anthropometry of particular segments might be unrealistic for a different population groups when using only scaling. So, personalization brings an additional value for improving the anthropometry of the particular dimensions of the particular subject, especially for pairing the human body with clothing, PPE and the external environment in general.

The purpose of the paper is to measure subject-specific anthropometric dimensions coming from the clothing industry for designing protective garments [15] for different population groups. The subject-specific dimensions are compared to the average anthropometry, which is obtained by scaling the reference anthropometry [16] using the average anthropometric data for the population group [17]. Based on the measured dimensions, a personalization algorithm for creating a subject specific human body model using only the clothing industry dimensions is developed.

There are also other initiatives [18] and options (e.g. 3D scanning) to generate a subject-specific model using three-dimensional scanning, however, the paper benefits from the existing scalable validated biomechanical virtual human body and extends the scaling algorithm by the personalization to develop a mathematical model to be used for numerical simulations in Visual Performance Solution (VPS) [19].

\section{Materials and Methods}

\subsection{Participants}

Four population groups including adults, teens and children were identified to show the variations in the particular anthropometric dimensions taking the territorial difference into account. The anthropometric measurements were carried out on Czech adults, employees of the University of West Bohemia, Czech children and Czech teens during sports activities. The total number of subjects was 152 ranging in age from 6 to 56 years. The Chinese adults, employees of the Tianjin Institute of Science and Technology, 
were carried out further. Their total number was 68 ranging in age from 24 to 60 years. The males and females were equally represented.

The measurements were carried out on adult students and adult employees $(\mathrm{N}=$ 70, of which 38 males and 32 females, age between 20 and 56 years, weight between 47 and 105 kilograms and height between 159 and 193 centimeters) from the Faculty of Applied Sciences, the Faculty of Education and the New Technologies - Research Centre, all three entities of the University of West Bohemia. Further, informed employees of the University of West Bohemia carried out the measurements on children $(\mathrm{N}=50$, of which 30 boys and 20 girls, age between 5.5 and 7.5 years, weight between 18.3 and 35.6 kilograms and height between 110 and 134 centimeters) from kindergartens cooperating with the Faculty of Education of the University of West Bohemia, and on teens $(\mathrm{N}=$ 32, of which 12 males and 20 females, age between 10 and 19 years, weight between 35 and 90 kilograms and height between 144 and 188 centimeters) who were students or children of employees of the University of West Bohemia, during sports courses or at home. Additional measurements were carried out on adult employees of the Tianjin Institute of Mechanical and Electrical Technology $(\mathrm{N}=68$, of which 33 males and 35 females, age between 24 and 60 years, weight between 49 and 110 kilograms and height between 155 and 184 centimeters) in China at their workplaces. Table 1 summarizes the measured subjects involved in the study.

Table 1. Subjects involved in the study. The number of participants and their age range (minimum $\wedge$ and maximum $\vee$ values).

\begin{tabular}{|c|c|c|c|c|}
\hline Gender & Czech children & Czech teens & Czech adults & Chinese adults \\
\hline Male & 38 & 30 & 12 & 33 \\
Female & 32 & 20 & 20 & 35 \\
All & 70 & 50 & 32 & 68 \\
\hline
\end{tabular}

\subsection{Anthropometric measurements}

The clothing industry anthropometric dimensions $[20,21]$ as shown in Figure 1 were measured using a tailor measuring tape on the particular body segments.

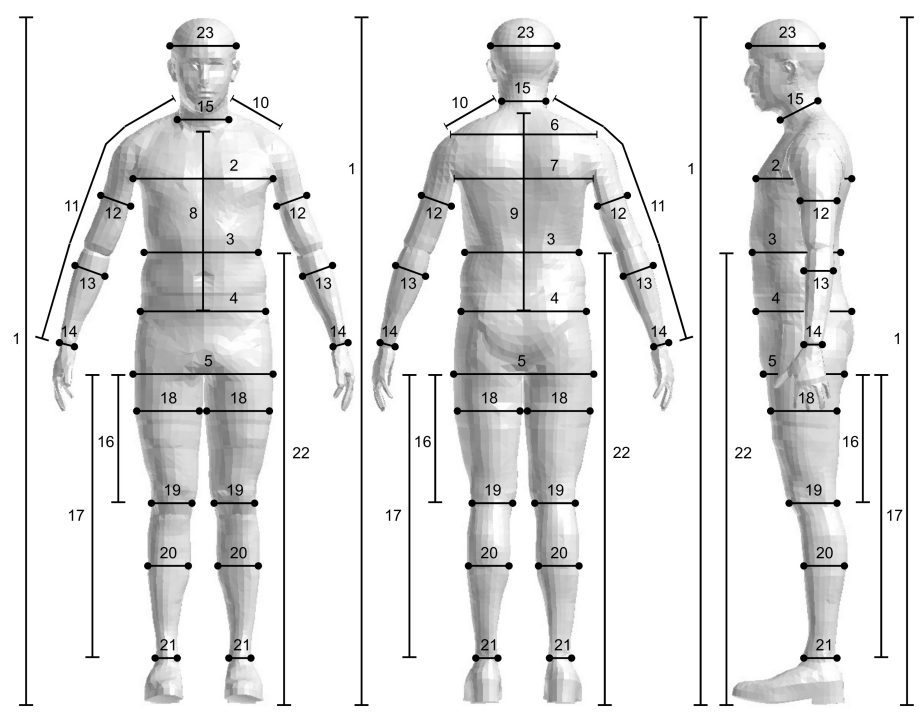

Figure 1. Clothing industry anthropometric dimensions. Identified clothing industry dimensions are adopted from the protective garment clothing industry [15].

The anthropometric dimensions are described in Table 2. The dimensions are mentioned by their number in the parenthesis in the further text. The age and the total weight of each subject were also reported. 
Table 2. Clothing anthropometric dimensions. Lengths between particular external body points and the circumference of particular segments.

\begin{tabular}{|c|c|c|}
\hline No. & Short name & Description \\
\hline 1 & Body height & Total body height \\
2 & Chest c. & Chest circumference (widest) \\
3 & Abdomen c & Waist circumference (narrowest) \\
4 & Waist c. & Waist circumference (trousers) \\
5 & Hip c. & Hip circumference (widest) \\
6 & Shoulder w. & Shoulder width \\
7 & Back w. & Back width \\
8 & F. thorax h. & Front length from neck to waist \\
9 & B. thorax h. & Back length from neck to waist \\
10 & Shoulder l. & Length from neck to shoulder \\
11 & Arm l. & Sleeve length from neck to wrist \\
12 & Arm c. & Arm circumference \\
13 & Forearm c. & Forearm circumference \\
14 & Wrist c. & Wrist circumference \\
15 & Neck c. & Neck circumference \\
16 & Thigh l. & Length from crotch to knee \\
17 & Leg l. & Length from crotch to ankle \\
18 & Thigh c. & Thigh circumference \\
19 & Knee c. & Knee circumference \\
20 & Calf c. & Calf circumference (widest) \\
21 & Ankle c. & Ankle circumference \\
22 & Abdomen h. & Length from waist to ground \\
23 & Head c. & Head circumference \\
\hline & &
\end{tabular}

The total body height (1) is the distance between the top of the head and the bottom of the heel when the subject stands upright. The measurements of the clothing industry dimensions of the particular body segments concern the torso, the head-neck area and the extremities. The chest circumference (2) is the circumference on the horizontal plane at the widest part of the thorax. The abdominal circumference (3) is the circumference on the horizontal plane at the narrowest part of the waist. The waist circumference (4) is measured on the horizontal section of the trousers. The hip circumference (5) is the circumference on the horizontal plane at the widest part of the hips.

The abdominal height (22) is the distance from the waist circumference to the ground. The shoulder width (6) is the distance between the shoulders equal to biacromial breath. The back width (7) is the thoracic width at the location of the chest circumference (2). The thoracic front length (8) is the distance from the neck bottom (suprasternal notch) to the horizontal plane of the abdominal circumference (3) on the front of the torso. The thoracic back length (9) is the distance from the C7 vertebra (vertebra prominens) on the horizontal plane of the abdominal circumference (3) on the back of the torso. The length from the neck to the shoulder (10) is the distance from the side neck bottom to the shoulder acromion.

The neck circumference (15) is measured around the middle section of the neck. In additional to the standard clothing industry dimensions, the head circumference (23) is measured on the horizontal plane a finger width above the eyebrow, as the head is a crucial body part to be protected and correctly anthropometrically modeled.

Concerning the upper extremities, the arm length (11) is the sleeve length from the neck to the location of the wrist circumference (14). The arm circumference (12) is the circumference around the widest biceps muscle and the forearm circumference (13) is the circumference around the widest forearm muscle. The wrist circumference (14) is measured at the wrist section.

Concerning the lower extremities, the thigh length (16) is the distance between the crotch and the horizontal plane of the knee section (19) and the leg length (17) is the distance between the crotch and the horizontal plane of the ankle section (21). 
The thigh circumference (18) is the circumference around the widest section of the quadriceps muscle. The knee circumference (19) is the circumference in the patella section. The calf circumference (20) is the circumference around the widest horizontal section of the calf muscle. The ankle circumference (21) is the circumference around the ankle section.

\subsection{Data analysis}

The subject-specific clothing industry dimensions were measured and analyzed for each of the four population groups including the Czech children, the Czech teens, the Czech adults and the Chinese adults. For each dimension, the minimum value, the mean value and the maximum value were calculated. The standard deviation for assessing the variation within the group completed the analysis.

Each subject-specific dimension was compared to its counterpart in the average anthropometry for the particular population group, which is obtained by scaling the reference model to the particular subject. The scaling of the reference model to the subject-specific human body model was based on gender, age, height and weight using the previously developed and published algorithm $[7,22]$.

The variation of the subject-specific dimensions from the average anthropometry in the particular population group was calculated as the average difference of all 23 clothing industry dimensions between the scaled anthropometry and the measured subject. The dimensions corresponding to the pair segments (the upper and the lower extremities) were addressed only once for the variation calculation.

\subsubsection{Reference model}

The reference model is a previously developed and published virtual biomechanical human body model [22] already implemented in a computational environment for assessing the risk of injury during an impact. Figure 2 shows the reference model representing an average human body based on the CEASAR database [16] corresponding to the hardware dummies Hybrid III and THOR-50M used for vehicle frontal crash testing [23].

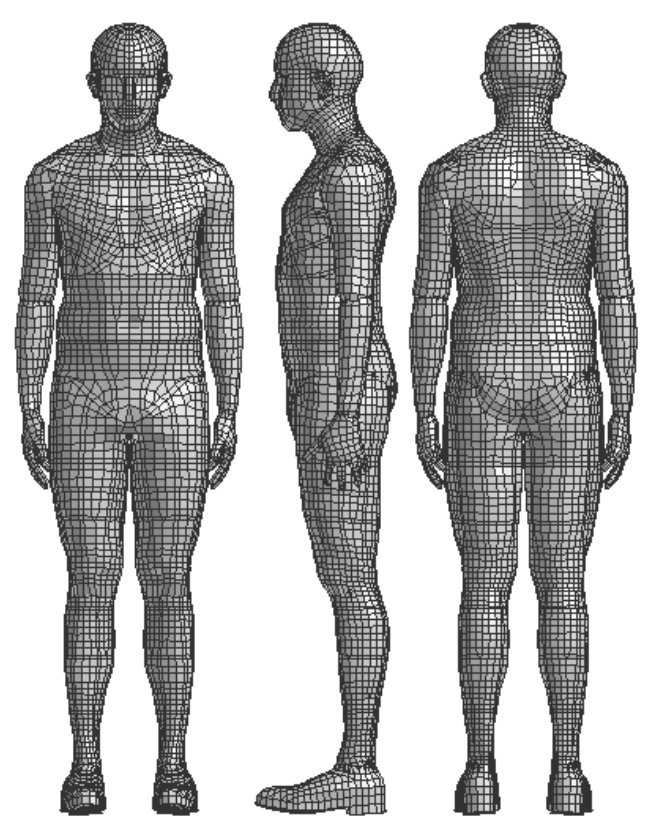

Figure 2. Reference model. Front (left), side (middle) and back (right) views. The reference model represents the average European anthropometry close to the Hybrid III 50 \% or Eurosid II dummies in terms of body dimensions. The reference model is a male with a weight equal to 77 $\mathrm{kg}$ and a height equal to $176 \mathrm{~cm}$ [24]. 
The model is hybrid as it couples the basic multi-body structure with deformable segments, performing in a short calculation time, and it is validated as being robust and biofidelic, meaning it converges for complex loading patterns (multi-directional) and has the correct mass distribution, stiffness and biomechanical behavior. As the dynamical simulation uses finite element discretization, each segment of the model is discretized by nodes forming elements to cover the external surface. Additionally, there are internal free nodes inside each segment to define the center of gravity, principal inertial axis and joint locations.

\subsection{Personalization}

The personalization method developed in the paper upgrades the existing scaling algorithm [7] by addressing the measured clothing industry dimensions to the firstly scaled reference body model shown in Figure 2 and so it develops a subject-specific virtual human body model. The algorithm benefits from the implementation of the clothing industry dimensions that are essential to design comfortable PPE. The presented personalization approach needs only basic subject-specific clothing industry dimensions, which are usually available and simply measurable. The external body shape is personalized using polynomial functions to secure the continuous and smooth body surface, which is desired mainly for applications where the human body comes into contact with other subjects or external objects.

\subsection{Personalization algorithm}

The personalization provides the local geometrical and biomechanical details of the human body. Firstly, the scaling [7] is done for each subject to target the given gender, age, height and weight. Having the scaled model, the personalization to provide correct anthropometric dimensions is carried out. The two-step approach is described as

$$
\begin{aligned}
T & =S+P \\
T(\mathbf{X}) & =S(\mathbf{X})+\Delta S(\mathbf{X}) \\
\mathbf{X}_{T} & =\mathbf{X}_{S}+\Delta \mathbf{X}_{S}
\end{aligned}
$$

meaning $T$ (target) equals $S$ (scaling) plus $P$ (personalization) where $\mathbf{X}_{T}$ describes the coordinates of any point of the subject-specific (target) model referring to the same point $\mathbf{X}$ in the reference model, which is firstly scaled to a semi-state $\mathbf{X}_{S}$ in the scaled model. We refer $\mathbf{X}$ to a spatial point in the global coordinate system with the origin in the H-point (the point in the middle between the hip joints) with the axis $x$ (defining the depth) pointing anteriorly to the front. The sagittal plane is the plane $x z$ (the axis $z$ defining the height points cranially to the head) and the axis $y$ (defining the width) directs laterally from the right to the left forming the right-hand side system.

Personalization is the next step after scaling. Scaling is a necessary step to have a model with global dimensions for the particular human body size before applying the mathematical operation to refine the surface locally. As the scaling algorithm was published previously, this paper will not address it, referring to the work by [7]. For personalization purposes, the scaled model is divided into segments summarized in Table 2 and schematically shown in Figure 3. Each segment can be described by the anthropometric dimensions from Table 2 displayed in Figure 1. The personalization algorithm runs independently of each of the 16 subject-specific segments defined in Table 3. 
Table 3. Segment numbers. Numbering of segments for personalization algorithm equations.

\begin{tabular}{|c|c|c|}
\hline Segment & Left & Right \\
\hline Head & \multicolumn{2}{|c|}{3} \\
Neck & \multicolumn{2}{|c|}{2} \\
Thorax & \multicolumn{2}{|c|}{1} \\
Abdomen & \multicolumn{2}{|c|}{0} \\
Arm & 4 & 7 \\
Forearm & 5 & 8 \\
Palm & 6 & 9 \\
Thigh & 10 & 13 \\
Calf & 11 & 14 \\
Foot & 12 & 15 \\
\hline
\end{tabular}

using the corresponding dimensions from Table 2 . There is a reference node $\mathbf{X}_{S 0}^{s}$ chosen on each segment as a local coordinate system origin, against which it is personalized, and which also serves to connect the neighboring segments following the open-tree segment system in the multi-body structure. It is usually the joint connecting the neighboring segments [22]. The personalization benefits from the open-tree structure hierarchy, so the base body (to be personalized first) is the abdomen followed by the thorax, neck and head upwards and the left and right thighs, the left and right calves and the left and right feet downwards. The thorax is followed by the left and right arms, left and right forearms and left and right palms. The reference model stands upright so that the local coordinate systems of all segments are aligned to the global coordinate system. The geometry and shape of a particular segment is given by the reference model, see Figure 2 .
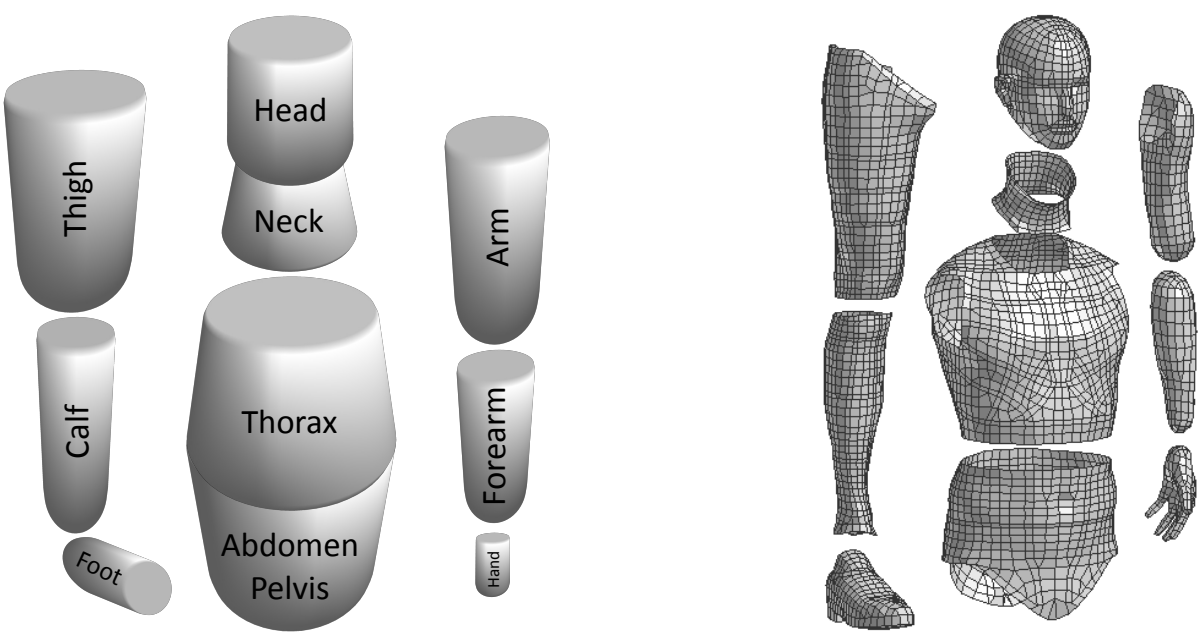

Figure 3. Human body segmentation. Methodology (left) and corresponding segmentation of reference model (right).

Any node $\boldsymbol{X}_{S i}^{s}, i \in\{1, \ldots, m\}$ ( $m$ being the numbers of nodes on the segment $s$ ) on the scaled model $S$ is personalized as

$$
\mathbf{X}_{T i}^{S}=\mathbf{R}^{S}\left(\mathbf{X}_{S i}^{S}-\mathbf{X}_{S 0}^{S}\right)+\mathbf{X}_{S 0}^{S}
$$

where $\mathbf{X}_{T i}^{s}$ is the personalized node on target model $T$ and $\mathbf{R}^{s}=\mathbf{R}^{s}(\mathbf{X})$ is the transformation matrix for particular segment $s$ depending on the spatial node position $\mathbf{X}$. Although the personalization in Equation 2 holds for all segments, the transformation matrix $\mathbf{R}^{s}$ contains the local scaling (personalization) coefficients to personalize particular segment $s \in\{0, \ldots, 15\}$ numbered according to Table 3 . For each segment $s \in\{0, \ldots, 15\}, \mathbf{R}^{s}$ is a diagonal matrix with the space dependent personalization coefficients in the global coordinate system, so that 


$$
\mathbf{R}^{s}=\left[\begin{array}{ccc}
p_{x}^{s}(\mathbf{X}) & 0 & 0 \\
0 & p_{y}^{s}(\mathbf{X}) & 0 \\
0 & 0 & p_{z}^{s}(\mathbf{X})
\end{array}\right]
$$

where $p_{i}^{s}$ for $i \in\{x, y, z\}$ are the personalization coefficients at location $\mathbf{X}$ in the global coordinate system for segment $s$. We use polynomials of degree $n_{i}^{s}$ in the form

$$
p_{i}^{\varsigma}(i)=\sum_{k=0}^{n_{i}^{s}} c_{i k}^{s} i^{k}=P_{i}^{s}\left(D_{j_{1}}, \ldots, D_{j_{n_{s}+1}}\right)
$$

to interpolate the personalization coefficient along each axis $i \in\{x, y, z\}$. Lower index $j_{r}$ for $r \in\left\{1, \ldots, n_{s}+1\right\}$ is chosen from set $\{1, \ldots, 23\}$ as numbered according to Table 2 . Vector $\mathbf{c}_{i}^{s}=\left[c_{i 0}^{s}, \ldots, c_{i n}^{s}\right]$ contains the polynomial coefficients for segment $s \in\{0, \ldots, 15\}$ in direction $i \in\{x, y, z\}$. Generally, indication $P\left(D_{j_{1}}, \ldots, D_{j_{n+1}}\right)$ means the interpolation polynomial of degree $n$ interpolating personalization coefficient as the ratios of $n+1$ anthropometric dimensions (see Figure 1) as

$$
D_{j}=\frac{(j)_{T}}{(j)_{S}}
$$

where lower indexes $T$ and $S$ mean target (personalized) and scaled dimensions, respectively, and $j \in\{1, \ldots, 23\}$ is the dimension number as numbered according to Table 2 and shown in Figure 1. For simplification in the further text, we indicate the personalization coefficient $D_{j}=j$. Particularly for example 15 means the neck circumference and 17-16 means the calf height (as it must be calculated by subtracting the thigh length from the leg length). For example, polynomial P(23) indicates a constant polynomial calculated as the ratio

$$
\frac{(23)_{T}}{(23)_{S}}
$$

polynomial $\mathrm{P}(17-16)$ indicates a constant polynomial calculated as the ratio

$$
\frac{(17)_{T}-(16)_{T}}{(17)_{S}-(16)_{S}}
$$

polynomial $\mathrm{P}(13,14)$ indicates a linear polynomial interpolating the personalization coefficient between points

$$
\frac{(13)_{T}}{(13)_{S}}, \quad \frac{(14)_{T}}{(14)_{S}}
$$

and polynomial $\mathrm{P}(6,7,3,4,5)$ indicates a polynomial of degree 4 interpolating the personalization coefficient between points

$$
\frac{(6)_{T}}{(6)_{S}}, \quad \frac{(7)_{T}}{(7)_{S}}, \quad \frac{(3)_{T}}{(3)_{S}}, \quad \frac{(4)_{T}}{(4)_{S}}, \frac{(5)_{T}}{(5)_{S}} .
$$

The polynomial interpolation might suffer from inaccuracy and unrealistic overshoots in the polynomial shape if we interpolate incomparable values, which is why the scaling is carried out in the first step to approach the target shape, which is refined by personalization.

For each segment, we firstly personalize the height based on the vertical dimensions (1), (8), (9), (10), (11), (16), (17) and (22) as shown in Figure 1 by means numbering according to Table 2 . The height of each segment is personalized constantly, meaning that

$$
p_{z}^{s}(\mathbf{X})=P\left(D_{j}\right)
$$


is the constant personalization coefficient for the particular segment height.

The frontal (segment depth) and lateral (segment width) personalization depends on the circumference dimension of the particular segment. The circumference dimensions are (2), (3), (4), (5), (12), (13), (14), (15), (18), (19), (20), (21) and (23) as shown in Figure 1 by means of numbering according to Table 2 . As many segments are described by circumference at the different horizontal location, the polynomials of the degree $n>0$ interpolate the personalization coefficient along axes $x$ and $y$ depending on axis $z$, which leads to the exact circumferences according to Figure 1 in the personalized model, so

$$
p_{i}^{s}=P_{i}^{s}\left(D_{1}, \ldots, D_{n_{s}+1}\right)
$$

where $i \in\{x, y\}$ and $n_{s}$ is the polynomial degree. We use the same polynomial degree $n^{s}$ for axes $x$ and $y$ for segment $s$, because the polynomials are formed by the same number of circumference dimensions for each segment.

Figure 1 also defines additional width dimensions, particularly (6) and (10), which are useful as additional measures for personalization. The personalization of each segment is described in detail in the following paragraphs.

\subsubsection{Abdomen}

The abdomen is the reference segment to be personalized first after the scaling. The reference point is the H-point. According to Figure 1, the height is personalized by a constant coefficient calculated as the ratio (between the target and scaled models) of the abdominal height (22), from which we subtract the length from the crotch to the ankle (17) and the ankle height (51). (51) is a new dimension, which is not addressed in the clothing industry, so we take it from the scaled model. As the abdomen and the thorax form a continuous segment, the polynomials interpolating the personalization coefficient in depth and width are formed together along the abdomen and the thoracic height. They are of degree 4 as they address 5 circumference dimensions. The depth is personalized along axis $z$ from the hip circumference at the widest point (5) over the waist circumference at the point of trousers (4), the waist circumference at the narrowest point (3), the thoracic circumference at the widest point (7) to the neck circumference (15), where the neck circumference seems to be a good measure for the upper thoracic depth. The width is personalized along axis $z$ from the hip circumference at the widest point (5) over the waist circumference at the point of trousers (4), the waist circumference at the narrowest point (3), the back width (7) to the shoulder width (6).

\subsubsection{Thorax}

The thorax follows the abdomen at the joint between vertebrae T12 and L1 [20], which is also the reference point. As the thorax height is defined by 2 dimensions in the sternum and on the back, the height is personalized linearly between the front length from neck to waist (8) and the back length from neck to waist (9) according to Figure 1. The depth and width are personalized using the same polynomials as for the abdomen.

\subsubsection{Neck}

The neck follows the thoracic at the joint between vertebrae C7 and T1 [20], which is also the reference point. According to Figure 1, the height is personalized by a constant coefficient calculated as the ratio of the total body height without shoes (1), from which we subtract the back length from neck to waist (9) and the abdominal height (22). As height 1-9-22 is the total height of the neck and the head, the same personalization coefficient is used for the head height. The depth is personalized by a constant coefficient using the neck circumference (5). The width is personalized along axis $z$ from the bottom neck using the neck circumference (15) over the neck circumference (15) to the head, where the personalization coefficient holds the neck circumference ratio (15). 62 is a new dimension, which is not addressed in the clothing industry, so we take it as the double shoulder length (10) subtracted from the back width (6). 


\subsubsection{Head}

The head follows the neck at the clivus [20]. As height 1-9-22 is the total height of the neck and the head, the same personalization coefficient is used for the head height. The height personalization uses the same personalization coefficient as the neck uses. The head size is not typically measured for the means of the clothing industry, so the coefficients are missing. Therefore, the depth and width are personalized by a constant coefficient using the head circumference (23), which is measured.

\subsubsection{Arm}

The arm follows the thorax in the shoulder joint [20]. The height (actually the length of the arm) is personalized using a constant coefficient defined by subtracting the shoulder length (10) from the sleeve length from neck to wrist (11). The depth is personalized by a parabolic polynomial based on the arm circumference (12). The polynomial holds the arm circumference ratio (12) in the elbow and the thoracic width ratio (6) at its height, which brings a realistic shape of the personalized arm. The width is personalized by a constant coefficient based on the arm circumference (12).

\subsubsection{Forearm}

The forearm follows the arm at the elbow joint [20]. The height personalization uses the same personalization coefficient as the arm uses. The depth and width are personalized using the same linear polynomials interpolating the ratios between the forearm circumference (13) and the wrist circumference (14).

\subsubsection{Palm}

As the palm is not typically measured in the clothing industry and its small size, mass and inertial effects do not influence any dynamical action considerably, the palm is not personalized and only the dimensions raising from of the scaling are taken into account.

\subsubsection{Thigh}

The thigh follows the abdomen in the hip joint [20]. According to Figure 1, the height is personalized by a constant coefficient addressing the ratio of the length from crotch to knee (16). The depth and width are personalized using the same cubic polynomials interpolating the ratios between the hip circumference at the widest point (5), the thigh circumference (18) and the knee circumference (19).

\subsubsection{Calf}

The calf follows the thigh in the knee joint [20]. The height is personalized by a constant coefficient defined by subtracting the length from the crotch to the knee (16) from the length from the crotch to the ankle (17). The depth and width are personalized linearly using the same polynomials interpolating the ratios between the calf circumference (20) and the ankle circumference (21).

\subsubsection{Foot}

The foot follows the calf in the hip ankle joint [20]. As the foot is not typically measured in the clothing industry and its small size, mass and inertial effects do not considerably influence any dynamical action, the foot is not personalized and only the dimensions raising from of the scaling are taken into account.

As the human body is expected to be symmetric, the personalization of left and right arms, forearms, palms, thighs, calves and feet is the same. Table 4 summarizes the personalization of all segments using the polynomial interpolation of the personalization coefficients along coordinate axes. 
Table 4. Personalization coefficients of particular segments. The numbers in brackets denote the particular dimension.

\begin{tabular}{|c|c|c|c|c|c|c|c|c|c|c|}
\hline Segment & Abdomen & Thorax & Neck & Head & Arm & Forearm & Palm & Thigh & Calf & Foot \\
\hline Height & $\mathrm{P}(22-17-51)$ & $\mathrm{P}(8,9)$ & $\mathrm{P}(1-9-22)$ & $\mathrm{P}(1-9-22)$ & $\mathrm{P}(11-10)$ & $\mathrm{P}(11-10)$ & 1 & $\mathrm{P}(16)$ & $\mathrm{P}(17-16)$ & 1 \\
Depth & $\mathrm{P}(5,4,3,7,15)$ & $\mathrm{P}(5,4,3,7,15)$ & $\mathrm{P}(5)$ & $\mathrm{P}(23)$ & $\mathrm{P}(72,12,12)$ & $\mathrm{P}(13,14)$ & 1 & $\mathrm{P}(5,18,19)$ & $\mathrm{P}(20,21)$ & 1 \\
Width & $\mathrm{P}(5,4,3,52,6)$ & $\mathrm{P}(5,4,3,52,6)$ & $\mathrm{P}(62,15,15)$ & $\mathrm{P}(23)$ & $\mathrm{P}(12)$ & $\mathrm{P}(13,14)$ & 1 & $\mathrm{P}(5,18,19)$ & $\mathrm{P}(20,21)$ & 1 \\
\hline
\end{tabular}

As the reference human body model is developed for dynamical analysis, the last step is updating the masses and inertias of the particular segments concerning the shape change as well as the ranges of the particular joints concerning the age using the same approach as described and published by [7].

The personalization process is implemented in Python. The Python script first reads the structure of the reference model as the finite element model [22]. The structure concerns the multi-body system, which separated the particular segments and their nodes representing the geometry into rigid bodies. The next step is the scaling of nodal coordinates and updating the stiffness of the joints, which is is updated independently using the approach previously developed and published $[7,25]$.

After the scaling of each segment, the personalization runs. Then, the segment masses and inertia are re-calculated based on the particular segment volume and shape change using the approaches previously developed and published [7]. After that, the segments are connected back together, as the volume and shape changes affected the location of joints. Finally, the new model is saved using the updated nodal coordinates, rigid bodies and joints. The joint stiffness is updated in parallel. The elements defining the body surface are kept as they follow the change of their nodal coordinates. The process flowchart is as follows:

1. Read reference model.

2. Scale reference model.

(a) Scale nodal coordinates [7].

(b) Update joint stiffness $[7,25]$.

3. Personalize scaled model.

(a) Personalize nodal coordinates according to Equation 2.

(b) Re-calculate mass and inertia [7].

4. Save personalized model.

The optimization loop is illustrated in Appendix A as a flowchart.

\section{Results}

The clothing industry dimensions for the four groups of Czech children, Czech teens, Czech adults and Chinese adults were measured and analyzed. Besides the clothing industry dimensions, the age $(a)$ in years and the total weight $(m)$ in kilograms of each subject were reported. The analysis concerned each particular population group (all) as well as the subgroups of males and females. Table 5 shows the mean values and the standard deviations, Table 6 shows the minimum and the maximum values. The standard deviation assesses the variation within the group. 
Table 5. Average dimensions. Mean value $(\oslash)$ and the standard deviation $(\sigma)$ for each dimension $(d)$. Average $(\oslash)$ and standard deviation $(\sigma)$ for each dimension. The age is reported in years, the mass is reported in kilograms and the length dimensions are reported in centimeters.

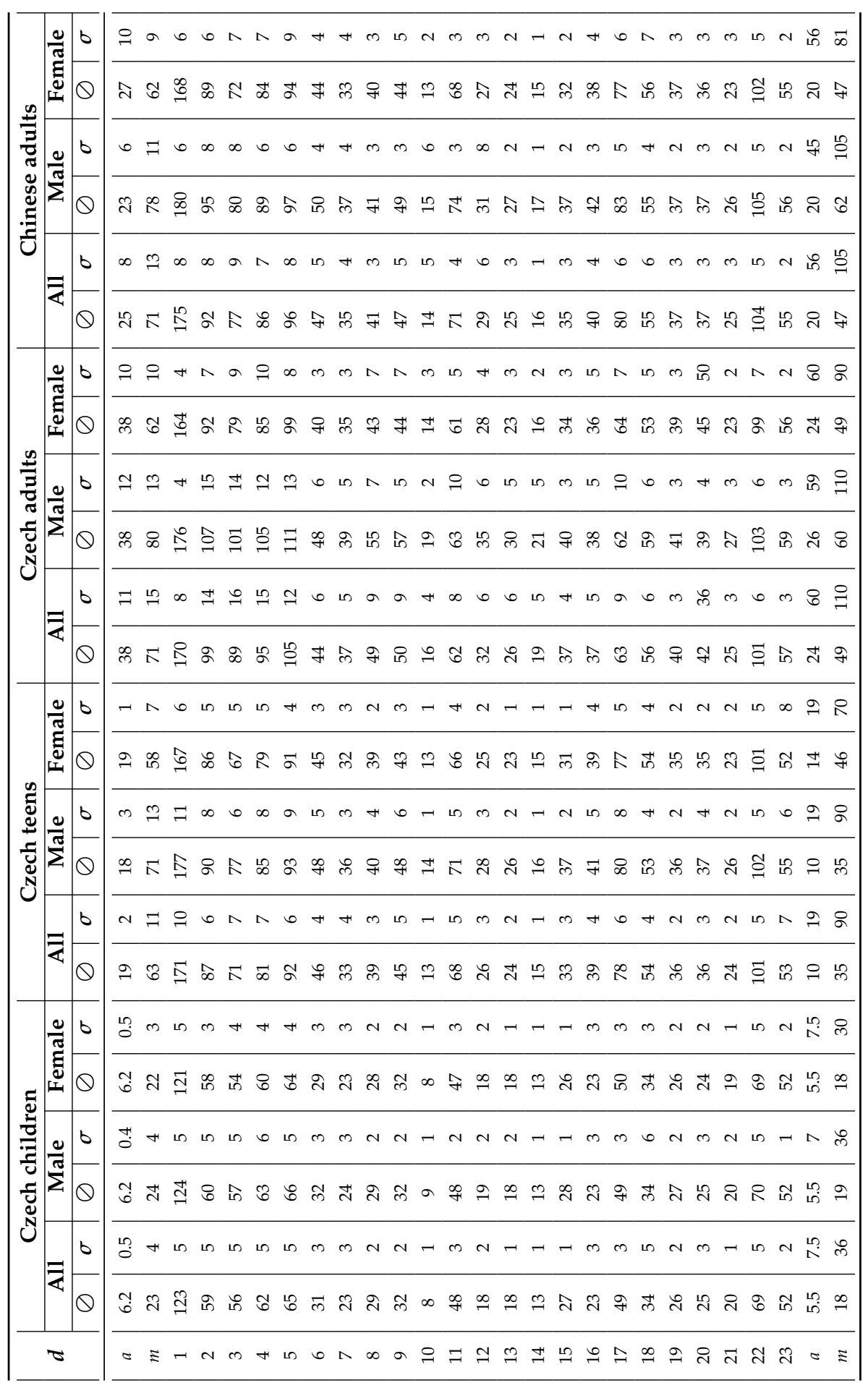


Table 6. Minimum and maximum values. Minimum $(\wedge)$ and maximum values $(\vee)$ for each dimension. The age is reported in years, the mass is reported in kilograms and the length dimensions are reported in centimeters.

\begin{tabular}{|c|c|c|c|}
\hline \multirow{6}{*}{ 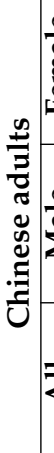 } & \multirow{2}{*}{\multicolumn{2}{|c|}{ 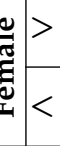 }} & 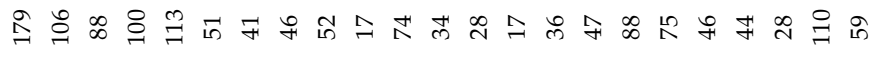 \\
\hline & & & ది \\
\hline & ص & $>$ & 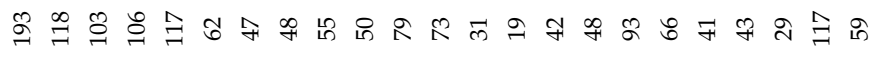 \\
\hline & $\Sigma$ & $<$ & 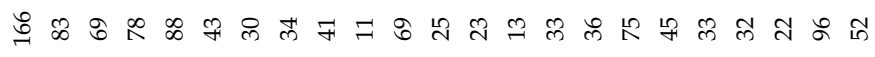 \\
\hline & & $>$ & 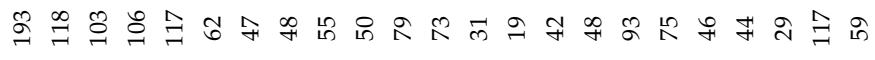 \\
\hline & & $<$ & 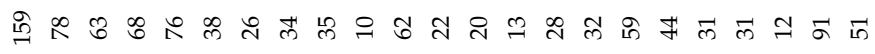 \\
\hline & $\frac{\varrho}{\tau} \mid$ & $>$ & 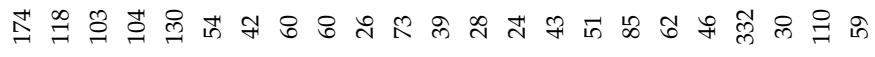 \\
\hline & $\mid$ & $<$ & 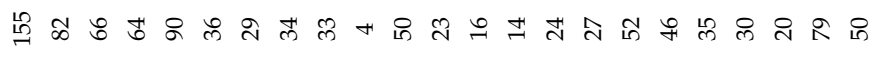 \\
\hline $\begin{array}{l}\bar{z} \\
\bar{z} \\
\end{array}$ & $\frac{\sim}{\pi}$ & $>$ & 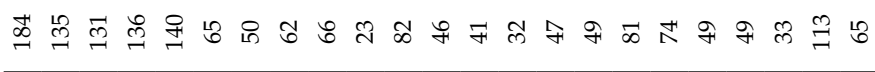 \\
\hline 总 & & $<$ & 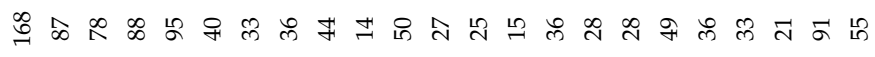 \\
\hline & & $>$ & 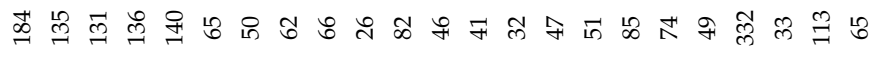 \\
\hline & & $<$ & 贵 $\infty$ \& \\
\hline & $\frac{\pi}{\pi}$ & $>$ & 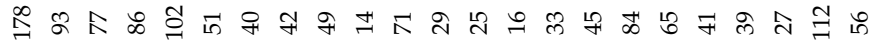 \\
\hline & $\mid \begin{array}{c}\bar{\omega} \\
\end{array}$ & $<$ & 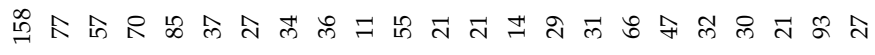 \\
\hline 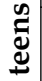 & $\underline{0}$ & $>$ & 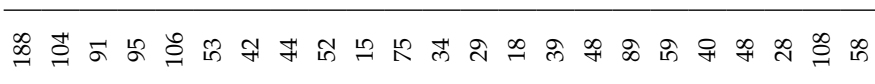 \\
\hline 芯 & $\Sigma$ & $<$ & 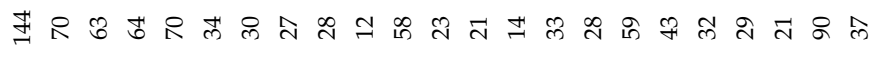 \\
\hline & & $>$ & 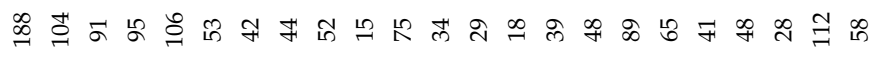 \\
\hline & & $<$ & Ұ尺 \\
\hline & $\frac{\varrho}{\Xi}$ & $>$ & స్తి $\overrightarrow{0}$ வ \\
\hline$\approx$ & 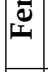 & $<$ & 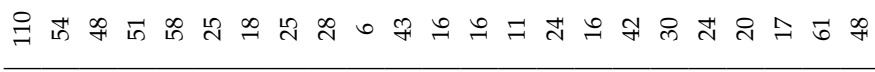 \\
\hline$\overline{\underline{z}}$ & 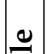 & $>$ & 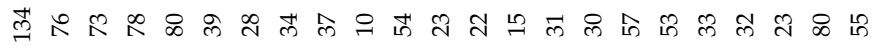 \\
\hline ปี & & $<$ & $\stackrel{\text { L }}{\Rightarrow}$ กิ \\
\hline u & - & $>$ & 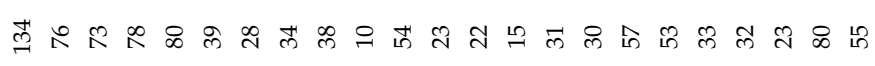 \\
\hline & & $<$ & 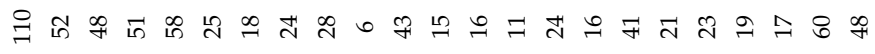 \\
\hline & $\tau$ & & 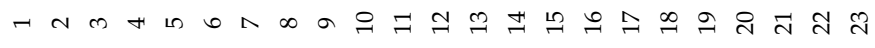 \\
\hline
\end{tabular}

All the measured subjects were reconstructed by scaling using the previously developed and published scaling algorithm [7] and the reference model [22]. The following figures compare the subject-specific dimensions obtained by scaling for different groups of subjects. Particular dimensions from Figure 1 are calculated based on the scaled reference model. The scaling variance is calculated as the average difference of all 23 
personalized dimensions between the scaled model and the measured subject. The pair segments are addressed only once.

The average variations and the standard deviations for the group of 70 Czech adults are displayed in Figure 4, the group of 32 Czech teens in Figure 5, the group of 50 Czech children in Figure 6 and the group of 68 Chinese adults in Figure 7.

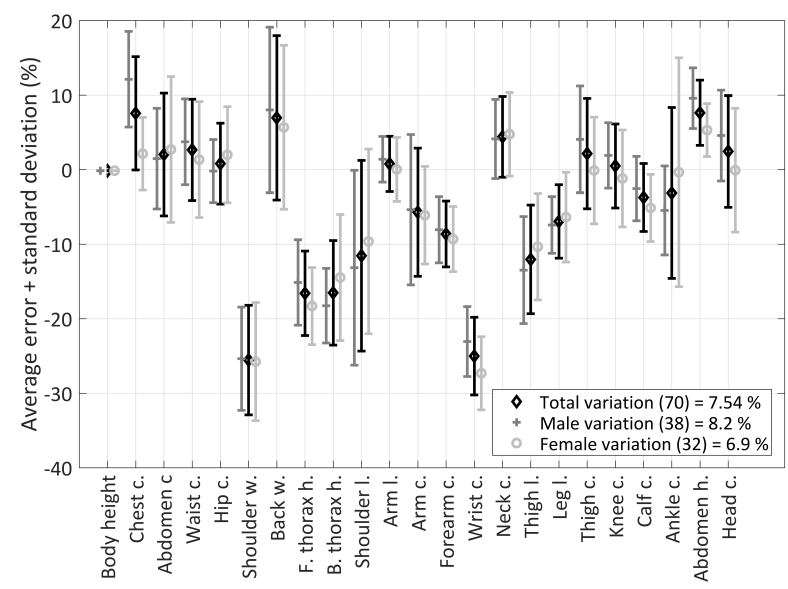

Figure 4. Czech adults. Average variations and the standard deviations.

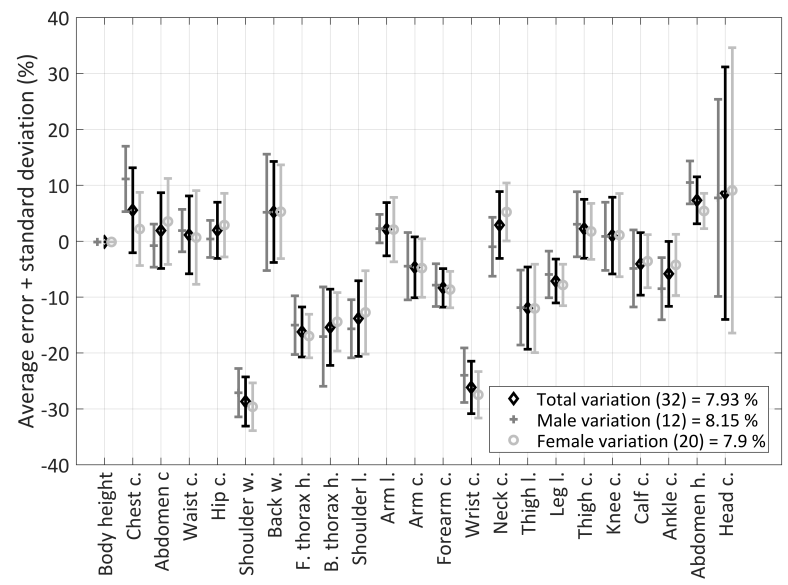

Figure 5. Czech teens. Average variations and the standard deviations.

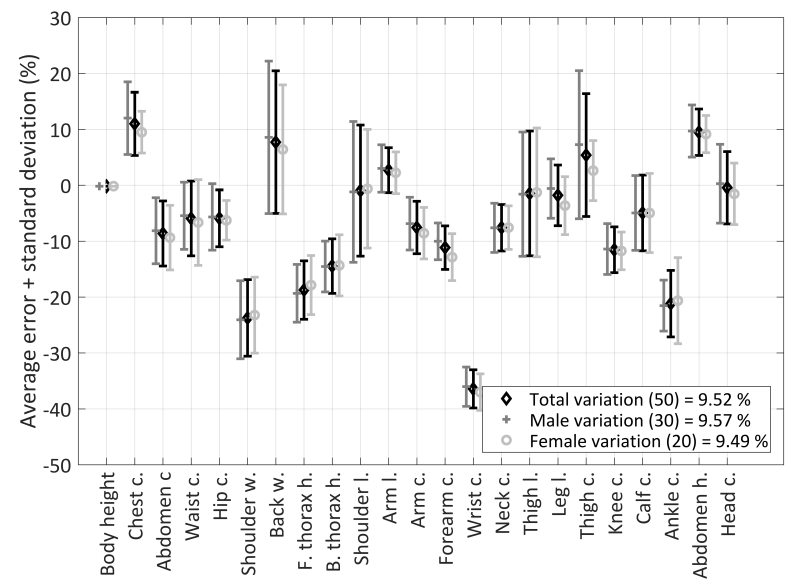

Figure 6. Czech children. Average variations and the standard deviations. 


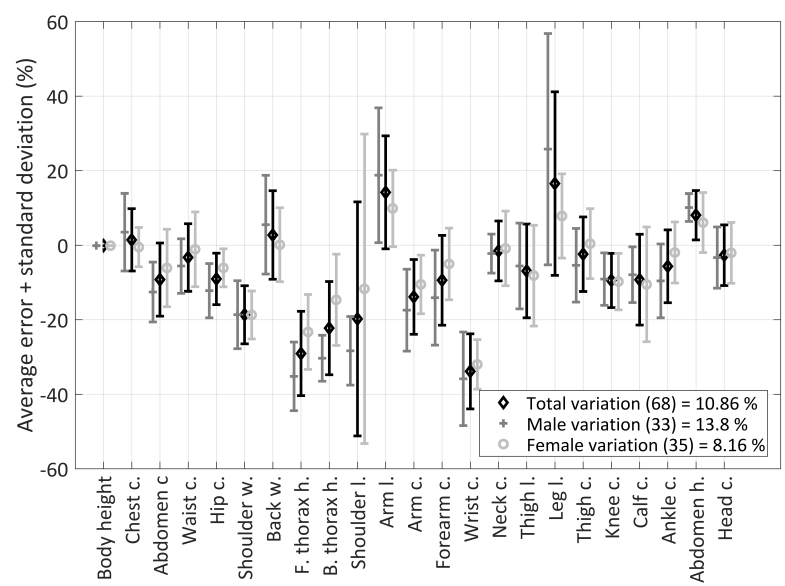

Figure 7. Chinese adults. Average variations and the standard deviations.

Based on the developed personalization algorithm, representatives of the particular population groups are displayed further. The personalization process is shown on Figure 8 using the model personalized in Figure 9. Firstly, the reference model shown in the left is scaled to became the scaled model, which is shown in the middle. The scaling algorithm updates the general dimensions and anthropometry, which is refined by the personalization leading to the correct model. Figure 9 concerns a 6-year old girl with a height of $118 \mathrm{~cm}$ and a mass equal to $19 \mathrm{~kg}$ from the Czech population group. Figure 10 concerns a 10-year old boy with a height of $144 \mathrm{~cm}$ and a mass equal to $35 \mathrm{~kg}$ from the Czech population group. Figure 11 concerns a 14-year old girl with a height of 160 $\mathrm{cm}$ and a mass equal to $46 \mathrm{~kg}$ from the Czech population group. Figure 12 concerns a 19-year old female with a height of $158 \mathrm{~cm}$ and a mass equal to $46 \mathrm{~kg}$ from the Czech population group. Figure 13 concerns a 45-year old male with a height of $176 \mathrm{~cm}$ and a mass equal to $85 \mathrm{~kg}$ from the Czech population group. Figure 14 concerns a 45 -year old female with a height of $164 \mathrm{~cm}$ and a mass equal to $63 \mathrm{~kg}$ from the Czech population group. Figure 15 concerns a 28-year old female with a height of $165 \mathrm{~cm}$ and a mass equal to $50 \mathrm{~kg}$ from the Chinese population group.

The displayed models are set to scale so that they can be compared with each other. By nature of the personalization algorithm targeting the particular dimensions from Table 2, the developed subject-specific virtual human body models fit to their real subject by those dimensions. 


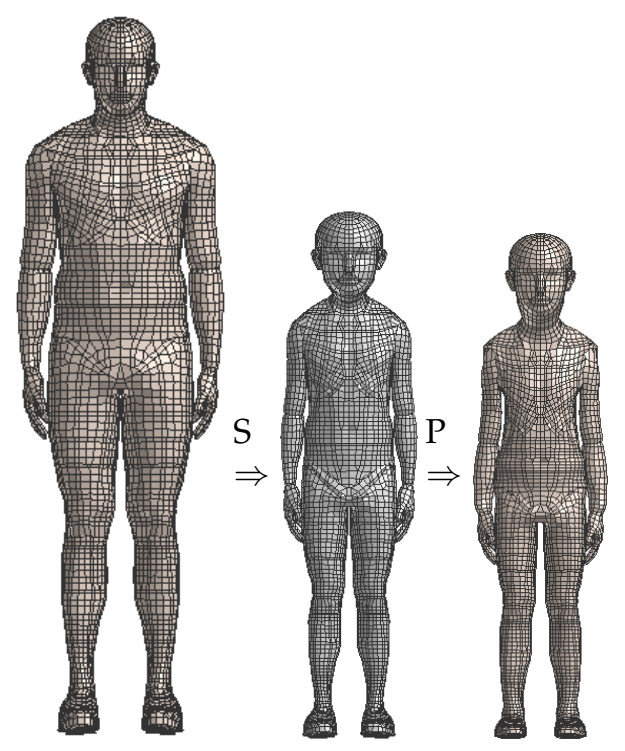

Figure 8. Personalization process. Reference model (left), scaled model (middle), personalized model (right).

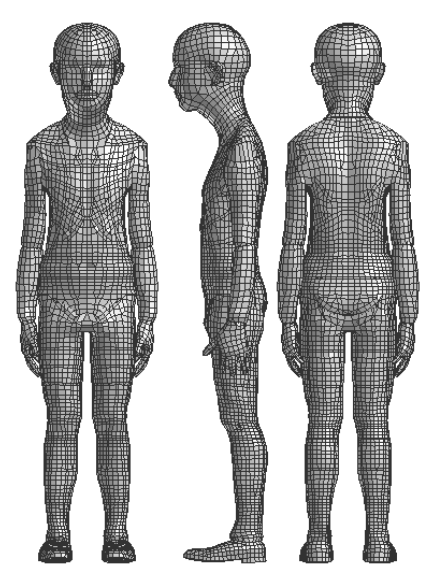

Figure 9. Subject-specific Czech child model. Female, 6 years, $118 \mathrm{~cm}, 19 \mathrm{~kg}$. Front (left), side (middle) and back (right) views.

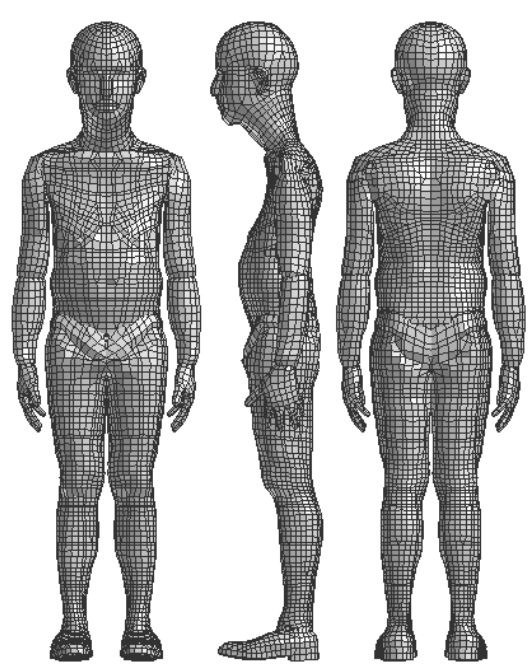

Figure 10. Subject-specific Czech teen model. Male, 10 years, $144 \mathrm{~cm}, 35 \mathrm{~kg}$. Front (left), side (middle) and back (right) views. 


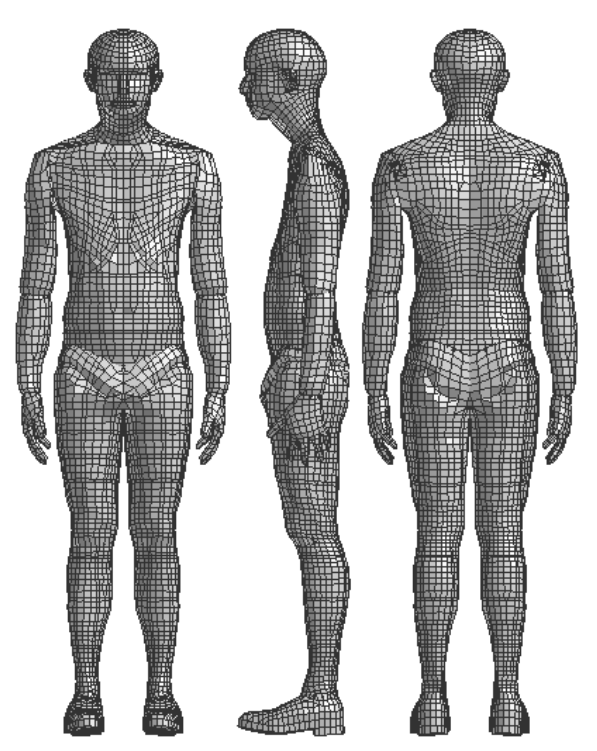

Figure 11. Subject-specific Czech teen model. Female, 14 years, $160 \mathrm{~cm}, 46 \mathrm{~kg}$. Front (left), side (middle) and back (right) views.

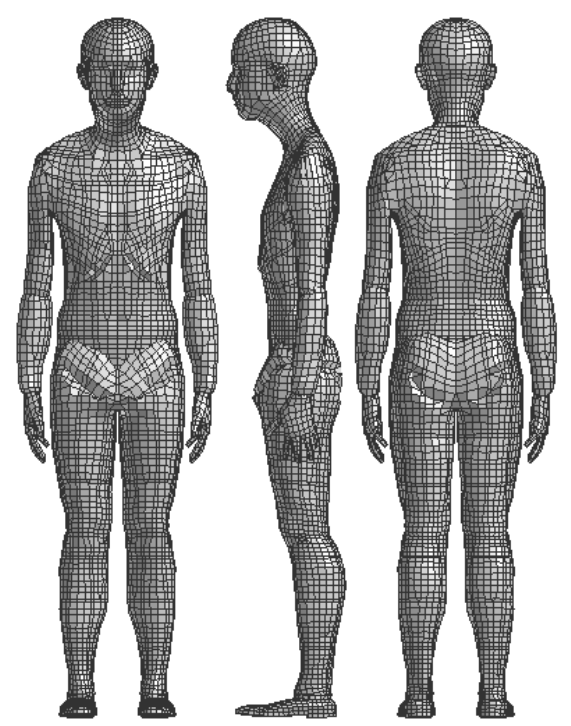

Figure 12. Subject-specific Czech teen model. Female, 19 years, $158 \mathrm{~cm}, 46 \mathrm{~kg}$. Front (left), side (middle) and back (right) views. 


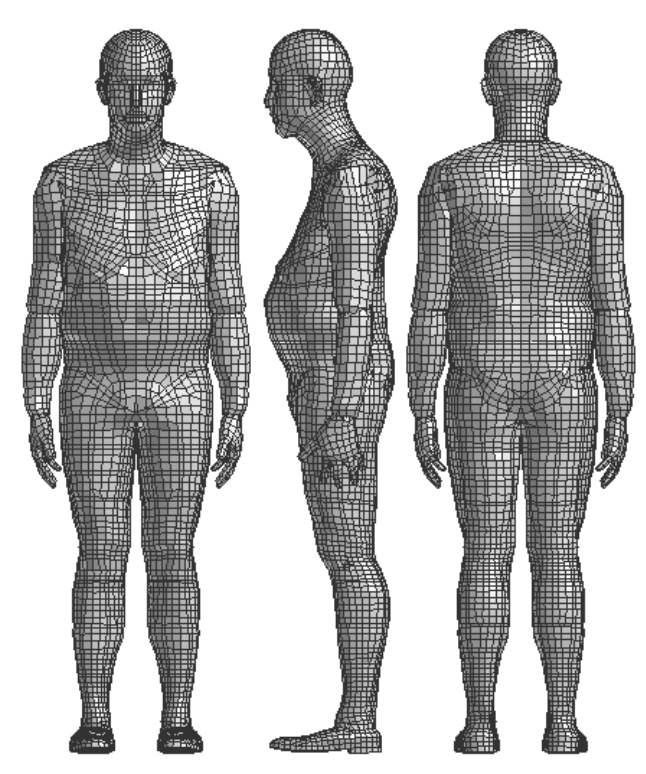

Figure 13. Subject-specific Czech adult model. Male, 45 years, $176 \mathrm{~cm}, 85 \mathrm{~kg}$. Front (left), side (middle) and back (right) views.

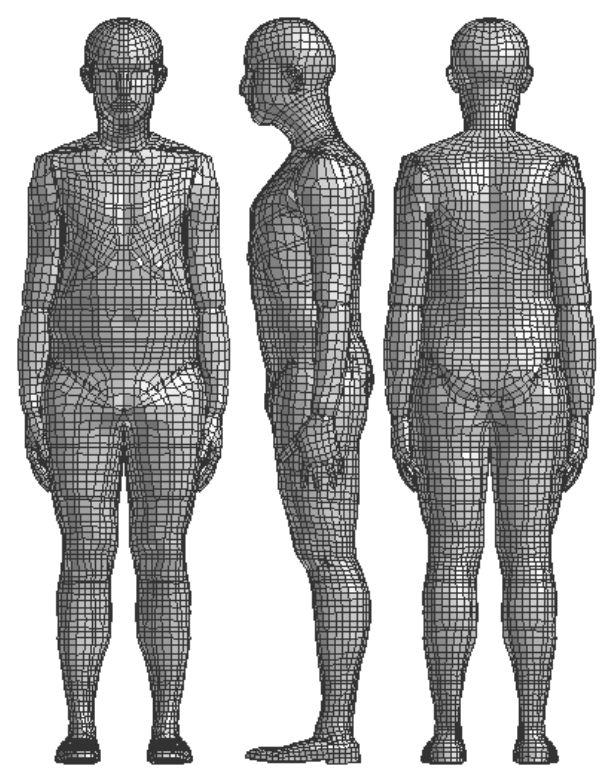

Figure 14. Subject-specific Czech adult model. Female, 45 years, $164 \mathrm{~cm}, 63 \mathrm{~kg}$. Front (left), side (middle) and back (right) views. 


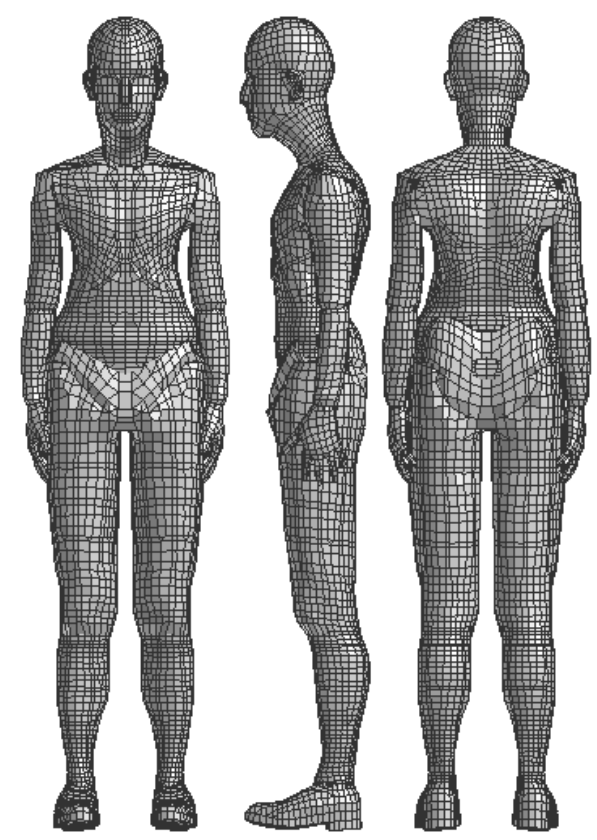

Figure 15. Subject-specific Chinese adult model. Female, 28 years, $165 \mathrm{~cm}, 50 \mathrm{~kg}$. Front (left), side (middle) and back (right) views.

\section{Discussion}

The presented study shows the variations of the subject-specific anthropometric dimensions from the average for different population groups in the Czech Republic and China as a background for the need for personalized human body models. The study shows variations in all dimensions except the total height, as it is one of the basic parameters for anthropometric modeling.

The variation of the Czech adults from the average (Figure 4 ) is the lowest in all groups and is around $7.54 \%$ ( $8.2 \%$ for males and $6.9 \%$ for females). This is probably caused by the fact that the reference model is an average European subject, which is close to the adult anthropometric group. The greatest variation is in the shoulder width (6) followed by the wrist circumference (14) and the frontal shoulder height (8). The great variation in the thoracic circumferences and the frontal thoracic height is probably caused due to the fact that the real shape distribution can be affected by the muscle mass or the breast size of the particular subject. The lowest variation is in the knee circumference (19) followed by the arm circumference (12) and the hip circumference (5) meaning that those dimensions can be well described by average anthropometry.

Decreasing the age, there is a slightly greater variation equal to $7.93 \%(8.15 \%$ for males and $7.9 \%$ for females) for the Czech teens (Figure 5). The teens, as well as the adults, are well described by the average European subject. The greatest variation here is again in the shoulder width (6), followed by the wrist circumference (14) and the frontal thoracic height (8). Again, all these measures can be affected by muscle mass. The lowest variation is in the knee circumference (19) followed by the waist circumference (4) and the abdominal circumference (3).

The average variation for the Czech children (Figure 6) equals $9.52 \%(9.57 \%$ for males and $9.49 \%$ for females). Here the variation is greater as the children have different anthropometry and segment proportions compared to the adults. However, the variation is not so great with the biggest in the wrist circumference (14) followed by the shoulder width (6) and the ankle circumference (21). The lowest variation is in the head circumference (23) followed by the shoulder length (10) and the thigh length (16).

The greatest average variation is seen in the group of Chinese adults (Figure 7) and is equal to $10.86 \%$ ( $13.8 \%$ for males and $8.16 \%$ for females). This average variation is even greater than in the case of the Czech children, meaning the different anthropometric 
proportions in the population. The standard deviation shows considerable differences even inside the group. The Chinese anthropometry is different from the European one, which causes the variation in dimensions average. The greatest variation is in the wrist circumference (14) followed by the frontal thoracic height (8) and the back thoracic height (9). The lowest variation is in the chest circumference (2) followed by the neck circumference (15) and the thigh circumference (16).

In general, for all groups, the variation is over the average for males, whilst females report variation under the average. This is probably caused by the anthropometric reference model, which represents both male and female groups, where males are usually more robust compared to females. The lowest variation is usually reached at dimensions which are not affected by the local musculature too much.

The personalized models, which provide the correct anthropometric dimensions, are based on the reference model surface mesh, where the problem of so-called downscaling occurs. This limitation of the study does not affect any dynamical calculation, however, the future development plans a remeshing of particular body parts. Another limitation of the study is that it focused only on limited groups in two territories. Even with such a limitation, the study proved the variation of the particular anthropometric dimensions, which might cause inaccuracy in virtual prototyping when developing PPE and passive safety measures.

\section{Conclusion}

The current paper provides a measurement of 23 major clothing industry dimensions on 220 human subjects including children, teens and adults in the Czech Republic and China. The number of Czech subjects was 152, ranging in age from 6 to 56 years. Additional measurements on 68 Chinese adults ranging in age from 24 to 60 years were carried out. The males and females were equally represented.

Using a single reference model, the subject-specific human body model is developed. On each subject, the scaling procedure was carried out to develop the generic scaled model related to the particular gender, age, height and weight. The anthropometric dimensions adopted from the clothing industry were compared to the measured ones. Measured subjects report variations in clothing industry dimensions when compared to an average subject of the same age. The cumulative variation of clothing industry dimensions increases the farer the population group from the average. The Czech subjects show lower variance than the Chinese subjects as the reference model is from the European database.

The study proves the variation of the particular anthropometric dimensions by comparison to the average anthropometric data, which might cause inaccuracies in virtual prototyping when developing PPE. Taking the results of the study into account, the development of personal protective systems should adopt the subject-specific models due to the variations from the average. So, the personalization algorithm to develop a subject-specific biomechanical human body model was developed and the chosen subjects from the anthropometric study are shown by means of their personalized models.

Scaling is a convenient tool for creating generic human body models representing a population group for virtual prototyping, such as developing safety systems in mobility or PPE. Modern technologies enable personalized products to be designed, so if we want to address a subject-specific approach for designing PPE, personalization refines the model towards the subject-specific anthropometry.

Author Contributions: Conceptualization, L.H.; methodology, L.H., G.K. and P.Š.; software, L.H. and H. Č; validation, L.H.; formal analysis, L.H., H.Č., T.B., G.K., P.Š and H.M.; investigation, L.H., T.B.,G.K., P.Š., V.H. and H.M.; resources, T.B.,G.K., P.Š., V.H. and H.M.; data curation, H.Č., T.B.,G.K., P.Š., V.H. and H.M.; writing-original draft preparation, L.H., H.Č., T.B., G.K. and P.Š; writing—review and editing, L.H., H.Č., T.B., G.K., P.Š, V.H. and H.M.; visualization, L.H.; 
supervision, L.H., G.K. and P.Š.; project administration, L.H.; funding acquisition, L.H. All authors have read and agreed to the published version of the manuscript.

Funding: This research was funded by the European Regional Development Fund-Project (grant number CZ.02.1.01/0.0/0.0/17_048/0007280) and by the University of West Bohemia Student Grant System (grant number SGS-2019-002).

Institutional Review Board Statement: The study was conducted according to the guidelines of the Declaration of Helsinki, and approved by the Ethics Committee of the New Technologies for Information Society of the Faculty of Applied Sciences of the University of West Bohemia (No. ZCU 011486/2017) from 23 January 2017, updated by Annex dated 29 April 2019, related to extending the approval for a consequent project.

Informed Consent Statement: Informed consent was obtained from each participant. In the case of the children, their legal representatives signed the informed consent as approved by the Ethics Committee.

Acknowledgments: The authors would like to thank all the subjects for their participation and their kind involvement in this study and the company PSí Hubík for providing samples and identifying dimensions of the personal protective garments.

Conflicts of Interest: The authors declare no conflict of interest.

\section{Abbreviations}

The following abbreviations are used in this manuscript:

PPE personal protective equipment

VPS Visual Performance Solution

\section{Appendix A. Iteration process flowchart}

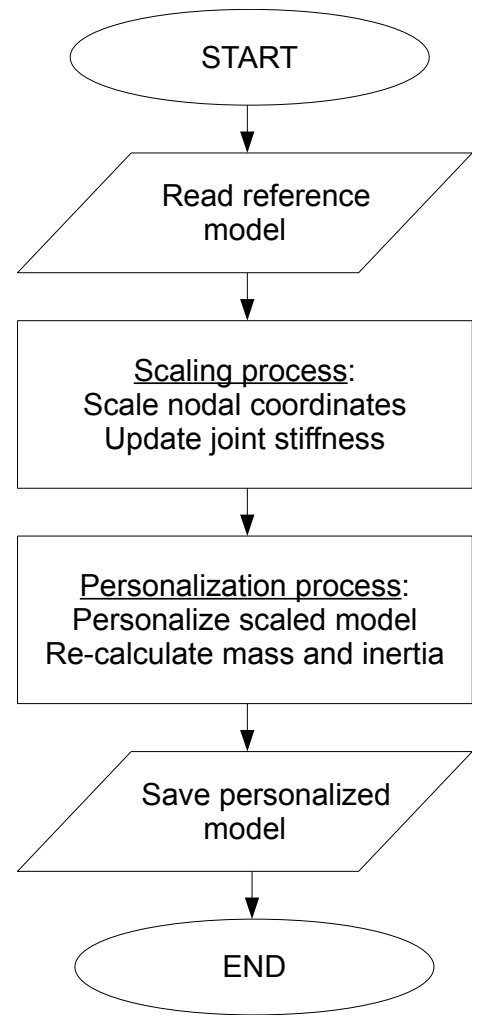

\section{References}

1. Gao, Y.; Xiajun, J. The Customized Human Body Modeling and Its Application in Damage Model Simulation. Asian Simulation Conference SCS Autumn Simulation Multi-Conference, 2016, pp. 1-11. doi:10.1007/978-981-10-2666-9_20. 
2. Koo, B.Y.; Park, E.J.; Choi, D.K.; Kim, J.J.; Choi, M.H. Example-based statistical framework for parametric modeling of human body shapes. Computers in Industry 2015, 72, 23-38. doi:10.4271/2020-01-0874.

3. Ayache, P.G. Computational Models for the Human Body; Vol. XII., Elsevier B. V., 2004.

4. Bońkowski, T.; Hynčík, L.; Šoltés, L. Motorcycle Helmets: The Population Diversity Influence on Head Injury Criterion Assessment. International Research Council on the Biomechanics of Injury Conference, 2017, pp. 1-2.

5. Bońkowski, T.; Hynčík, L.; Špička, J.; Vychytil, J. Motorcycle accidents reconstruction and simulation - -application of hybrid human body model. Mathematical Modeling 2021, 4 (3), 100-103.

6. Happee, R.; van Haaster, R.; Michaelsen, L.H.R. Optimisation of vehicle passive safety for occupants with varying anthropometry. ESV Technical paper 98-S9-O-03 1998, pp. 1-6.

7. Hynčík, L.; Čechová, H.; Kovář, L.; Bláha, P. On Scaling Virtual Human Models. SAE Technical Paper 2013-01-0074 2013, pp. 1-8. doi:10.4271/2013-01-0074.

8. Hynčík, L.; Špička, J.; Maňás, J.; Vychytil, J. Stature Based Approach towards Vehicle Safety. SAE Technical Paper 2015-26-0209 2015, pp. 1-7. doi:10.4271/2015-26-0209.

9. Hwang, E.; Hallman, J.; Klein, K.; Rupp, J.; al.. Rapid Development of Diverse Human Body Models for Crash Simulations through Mesh Morphing. SAE Technical Paper 2016-01-1491 2016. doi:10.4271/2016-01-1491.

10. Ma, L.; Zhang, K.; Lu, D. Research and application of personalized human body simplification and fusion method. EURASIP Journal on Image and Video Processing 2018, 55, 1-10. doi:10.1186/s13640-018-0293-7.

11. Liu, S.; Beillas, P.; Ding, L.; Wang, X. Morphing an Existing Open Source Human Body Model into a Personalized Model for Seating Discomfort Investigation. SAE Technical Paper 2020-01-0874 2020, pp. 1-8. doi:10.4271/2020-01-0874.

12. Alexa, M. Recent Advances in Mesh Morphing. Computer Graphics Formum 2002, 21, 173-197.

13. Reed, M.P.; Raschke, U.; Tirumali, R.; B., P.M. Parametric Human Body Models in Ergonomics Software. The $3^{\text {rd }}$ International Digital Human Modeling Symposium, 2014, pp. 1-8.

14. Sigal, I.A.; Hardistya, M.R.; Whynea, C.M. Mesh-morphing algorithms for specimen-specific finite element modeling. Journal of Biomechanics 2008, 41, 1381-1389. doi:10.1016/j.jbiomech.2008.02.019.

15. PSí Hubík. The ready-made sizes, 2020 (accessed 6 November 2020).

16. Robinette, K.; Blackwell, S.; Daanen, H.A.M.; Boehmer, M.; Fleming, S.; Brill, T.; Hoeferlin, D.; Burnsides, D. Civilian American and European Surface Anthropometry Resource (CAESAR), Final Report. Volume 1. Summary. Technical report, 2002.

17. Bláha, P. Anthropometry of Czech and Slovak Population from 6 till 55 years. Technical report, 1986.

18. Janák, T.; Lafon, Y.; Petit, P.; Beillas, P. A Method to Use Kriging With Large Sets of Control Points to Morph Finite Element Models of the Human Body. journal of Biomechanicsl Engineering 2020, 143, 1-17. doi:10.1115/1.4048575.

19. International, E.G. VPS User's Manual. ESI Group International, 2020.

20. Williams, P.L. Gray's Anatomy; Churchill Livingstone: London, United Kingdom, 1995.

21. Simmons, K.P.; Istook, C.L. Body measurement techniques: Comparing 3D body-scanning and anthropometric methods for apparel applications. Journal of Fashion Marketing and Management 2003, 7(3), 306-332. doi:10.1108/13612020310484852.

22. Vychytil, J.; Maňas, J.; Čechová, H.; Špirk, S.; Hynčík, L.; Kovář. Multi-Directional Virtual Human Model for Safety Assessment. SAE Technical Paper 2014-01-0534 2014, pp. 1-22. doi:10.4271/2014-01-0534.

23. Humanetics. Anthropomorphic Test Devices (ATD), 2021 (accessed 11 June 2021).

24. Bońkowski, T.; Hynčík, L.; Lv, W. PTW Passive Safety: Numerical Study of Standard Impact Scenarios with Rider Injury Risk Assessment. SAE Technical Paper 2020-01-0930 2020, pp. 1-25. doi:10.4271/2020-01-0930.

25. Araújo, C.G. Flexibility assessment: normative values for flexitest from 5 to 91 years of age. Arq. Bras. Cardiol. 2008, 90 (4), 257-263. doi:10.1590/S0066-782X2008000400008. 